\title{
Predictors of Postoperative Hypocalcemia after Total Thyroidectomy: The Role of a Single Intraoperative Rapid PTH
}

\author{
Marc Rafols, $M D^{1}$, Daniela Treitl, $M D^{1}$, Luis E Rosario, $M D^{1}$, Juan Paramo, $M D^{2}$, Luigi X Cubeddu $M D$, \\ Ph. $D^{3}$ and Thomas Mesko, $M D^{2 *}$
}

${ }^{1}$ Medical Education, Resident Physician, General Surgery, Mount Sinai Medical Center, Miami Beach, FL, USA

${ }^{2}$ Attending Physician, Surgical Oncology, Mount Sinai Medical Center, Miami Beach, FL, USA

${ }^{3}$ Professor, COP, HPD, COP, Nova Southeastern University, Ft. Lauderdale, FL, USA

\begin{abstract}
Background: Postoperative hypocalcemia (PoHC) is the most common complication of total thyroidectomy (TT). The availability of a reliable predictor of PoHC would allow early calcium supplementation, avoiding hypocalcemia, and potentially decreasing hospital stay.
\end{abstract}

Objective: To determine the value of a single rapid intraoperative PTH (iPTH) level and influence of patient demographics, thyroid pathology, and lymph node resection in predicting PoHC after TT.

Study design: A single-institution prospective observational study.

Subjects and methods: Ninety-eight patients underwent a total or completion TT over one year. PTH was measured intraoperatively 15 minutes after completion of the TT. Hypocalcemia was defined as corrected calcium levels of $\leq 7.5$ $\mathrm{mg} / \mathrm{dL}$ or symptomatic hypocalcemia. ROC-AUC and logist regression analysis of $\mathrm{PPTH}$, age, and BMI was conducted.

Results: Compared to normocalcemic patients $(n=76)$, hypocalcemic patients $(n=22)$ were younger $(44.8 \pm 3.1$ vs. 63.2 \pm 1.6 years; $p<0.0001)$, had lower BMI $(25.4 \pm 0.9$ vs. $29.1 \pm 0.6 \mathrm{~kg} / \mathrm{sqm} ; \mathrm{P}=0.0045)$ and lower iPTH levels $(15.5 \pm 2.4$ vs. $61.7 \pm 7.3 \mathrm{pg} / \mathrm{ml} ; \mathrm{P}=0.0033)$. Serum calcium levels were directly associated with younger age $(P=0.0004)$, lower $B M I(P$ $=0.0003)$, and lower iPTH levels $(P=0.0004)$. IPTH levels lower than $16.75 \mathrm{pg} / \mathrm{ml}$ (ROC-AUC: $0.825 ; 95 \% \mathrm{Cl} 0.74-0.93 ; \mathrm{p}<$ 0.0001 ) identified hypocalcemia with a sensitivity of $77 \%$ and a specificity of $75 \%$. Levels of iPTH $\geq 36.25 \mathrm{pg} / \mathrm{ml}$ predicted normocalcemia with a sensitivity of $95.5 \%$ and a PPV 97.8. The combination of $\mathrm{PPTH}$, age, and BMI further improved the risk prediction of PoHC (mean ROC-AUC 0.95; 95\% Cl: 0.91-0.99). A combination of iPTH > $12.5 \mathrm{pg} / \mathrm{ml}$, > 31 years of age, and $\mathrm{B} \mathrm{BMI}>22.5 \mathrm{~kg} / \mathrm{m} 2$ predicted a normocalcemic course with a sensitivity of $100 \%$. Lymph node dissection, histologic diagnosis, indication for surgery, and intraoperative complications were not associated with serum calcium, age, BMI, or iPTH levels.

Conclusion: A single low iPTH is strongly and significantly associated with PoHC in post-thyroidectomy patients. Age and $\mathrm{BMI}$ also independently predicted risk of PoHC. Younger age and lower BMI increased risk of PoHC and increased the predictive power of iPTH.

\section{Keywords}

Rapid intraoperative PTH, Thyroidectomy, Post-operative hypocalcemia, AGE, BMI, ROC, Predictors of hypocalcemia, Risk-stratification

\section{Introduction}

The occurrence of postoperative hypocalcemia (PoHC) after total thyroidectomy (TT) has been reported to range from $1.3 \%$ to $50 \%$ [1]. PoHC is believed to be caused by trauma or devascularization of the parathyroid glands or inadvertent removal of one or more parathyroid glands at the time of surgery [2,3]. Although most cases of PoHC are transient and subclinical, symptomatic $\mathrm{PoHC}$ requires treatment and can extend the hospital stay by up to $48-72$ hours [4-6]. Because PoHC often does not develop until 24-48 hours postoperatively [7], early predictors of PoHC would allow prompt management preventing $\mathrm{PoHC}$, and potentially reducing the length of hospital stay.

A single postoperative PTH level or decrease from preoperative PTH levels following TT has been employed to predict PoHC $[1,3]$. PTH levels have been shown to have a high sensitivity and specificity in predicting PoHC [4,8-10], whereas, 
Citation: Rafols M, Treitl D, Rosario LE, et al. (2020) Predictors of Postoperative Hypocalcemia after Total Thyroidectomy: The Role of a Single Intraoperative Rapid PTH. J Surgical Endocrinol 2(1):61-66

other investigators reported that postoperative PTH is not a reliable predictor of $\mathrm{PoHC}$ [11] finding no correlation between postoperative PTH and postoperative calcium [12]. Differences in sampling times are known to affect PTH's predictive value $[4,7,11,12]$. Beside PTH, patient characteristics, the surgical procedure employed, need for lymph node resection, and possible complications may also play a role in determining the risk of developing PoHC. Therefore, we investigated the value of a single PTH level, when obtained immediately (15 min) after completion of the $\mathrm{TT}$, and investigated the presence of additional independent risk factors that may improve the prediction of PoHC.

\section{Objectives}

The primary aim of this study was to determine the value of iPTH levels in predicting PoHC. Additionally, we investigated whether patient age, BMI, preoperative calcium levels, clinical scenario, tissue pathology, surgical procedure, lymph node resection, and surgery complications predicted PoHC, and improved the predictive value of $\mathrm{iPTH}$.

\section{Patient and Methods}

A single-center, open-label, prospective observational study was conducted from January 2016 to January 2017 in patients who underwent total or completion TT at Mount Sinai Medical Center, Miami Beach, FL. The following were the exclusion criteria: Advanced chronic kidney disease with a creatinine clearance of less than $40 \mathrm{ml} / \mathrm{min}$, abnormalities in calcium homeostasis, hyper or hypoparathyroidism, treatment with diuretics, hematologic malignant tumors with paraneoplastic syndrome, vitamin D deficiency, and calcium supplementation before post-operative calcium lab draw. Of the 103 eligible patients, two were excluded due to preoperative vitamin $D$ deficiency, and three due to early calcium supplementation. The remaining 98 patients were included in the study. Surgery was performed by either one of two of the authors of this study (TM or JP).

The following variables were recorded: Age, sex, BMI, ethnicity, comorbidities, preoperative calcium, and vitamin D levels, indication for surgery (benign, malignant, or other), type of surgery (completion vs. TT), whether any lymph node dissection was performed, length of stay, complications, and postoperative serum calcium, iPTH, and albumin levels. A venous sample was obtained $15 \mathrm{~min}$ after the $\mathrm{TT}$, while the patient was still in the operating room. The sample was obtained, by direct venipuncture of the internal jugular vein ipsilateral to the lobe that was dissected first. We chose the internal jugular vein because it is easily accessible at the time of thyroid surgery, and directly drains the parathyroid glands [3]. Briefly, samples were put in ice and transported immediately from the operating room to the programmed laboratory. There they were centrifuged at 3,500 rpm for 3 minutes at $4{ }^{\circ} \mathrm{C}$ and analyzed ( 15 minutes of instrument assay using the Siemens Immulite 2000 system). Results were attained while the patient was still in the recovery room.

A standardized post-thyroidectomy protocol was used in all patients undergoing a TT at our Institution. Baseline serum calcium and albumin levels were obtained in the recov- ery room. Serial calcium levels were obtained every 6 hours for a total of 48 hours. We defined biochemical hypocalcemia when the corrected serum calcium level $\left(\mathrm{Ca}^{2+}\right.$ corrected $=\mathrm{Ca}^{2+}$ serum $+(0.8$ [4 - albumin]) was less than $7.5 \mathrm{mg} /$ dl. Symptoms of hypocalcemia were defined as numbness or paresthesia in distal extremities, perioral numbness or paresthesia, carpopedal spasms, tetany, or positive Chvostek's sign. All patients that developed biochemical hypocalcemia or symptoms of hypocalcemia were accounted for. Symptoms of hypocalcemia and Chvostek sign were monitored during hospitalization every 6 to 8 hours. If the patient-reported symptoms consistent with hypocalcemia, additional serum calcium levels were obtained immediately at that time.

Results were expressed as the number of observations, means, SEM, and $95 \% \mathrm{Cl}$ when specified. The sensitivity and specificity, NPV, PPV of iPTH in predicting PoHC were calculated. Differences between means were analyzed with the Student's t-test. Differences between ratios and for categorical variables were analyzed with the Fisher's exact test. Receiver operator characteristics (ROC) area-under-the-curve (AUC) were used to define cut-off values with the greatest sensitivity and specificity. Correlation analyses and correlation matrix, univariate, and logistic regression analyses were performed with the SPSS vs. 26 software.

\section{Results}

98 patients participated in the study; 82 were women and 16 men (Table 1 ). The mean age was $59.0 \pm 1.64$ years. Of the surgeries performed 96 were TT, and 2 were completion thyroidectomies. 57 patients had benign disease, and 41 had thyroid cancer. 19 patients underwent lymph node dissections including, central, unilateral, or bilateral cervical lymph node dissection. 10 patients had substernal thyroid extension, but none required sternotomy. LOS averaged $32.4 \pm 1.7$ hours after surgery (Table 1 ).

Preoperative calcium levels averaged $9.45 \pm 0.06 \mathrm{mg} / \mathrm{dl}$, dropping an average of $1.29 \pm 0.118 \mathrm{mg} / \mathrm{dl}(15.7 \%)$ after TT (lowest calcium levels after TT) (Table 1). Twenty-two patients developed $\mathrm{PoHC}$, while 76 patients remained normocalcemic in the post-operative course. There were no significant differences in the preoperative levels of serum calcium of patients who developed PoHC or that had a normocalcemic course (Table 2). The average postoperative corrected calcium was $8.42 \pm 0.05 \mathrm{mg} / \mathrm{dl}$ for normocalcemic patients and $7.42 \pm 0.06$ $\mathrm{mg} / \mathrm{dl}$ for hypocalcemic patients $(\mathrm{p}<0.0001)$ (Table 2$)$. Of the

* Corresponding author: Thomas Mesko, MD. Assistant Professor, Department of Surgical Oncology, Mount Sinai Medical Center, 4306 Alton Road, $2^{\text {nd }}$ Floor, Miami Beach, FL, 33140, USA, Tel: 305-674-2397, Fax: 305-674-2863

Accepted: December 28, 2020

Published online: December 30, 2020

Citation: Rafols M, Treitl D, Rosario LE, et al. (2020) Predictors of Postoperative Hypocalcemia after Total Thyroidectomy: The Role of a Single Intraoperative Rapid PTH. J Surgical Endocrinol 2(1):61-66 
Citation: Rafols M, Treitl D, Rosario LE, et al. (2020) Predictors of Postoperative Hypocalcemia after Total Thyroidectomy: The Role of a Single Intraoperative Rapid PTH. J Surgical Endocrinol 2(1):61-66

Table 1: Demographics, biochemistry, indications for surgery, surgical intervention and tissue pathology of patients that underwent total or completion thyroidectomy.

\begin{tabular}{|c|c|}
\hline Number of patients & 98 \\
\hline Age & $59.0 \pm 1.6$ \\
\hline Gender F/M & $82 / 16$ \\
\hline BMI & $28.3 \pm 0.53$ \\
\hline \multicolumn{2}{|l|}{ Indication for Surgery } \\
\hline Biopsy proven malignancy & $18(18.3 \%)$ \\
\hline Goiter /compressive symptoms & $15(15.3 \%)$ \\
\hline Multinodular Goiter & $23(23.4 \%)$ \\
\hline Suspicious Nodule & $27(27.5 \%)$ \\
\hline Hyperthyroidism (toxic nodule) & $2(2.0 \%)$ \\
\hline Graves Disease & $15(15.3 \%)$ \\
\hline \multicolumn{2}{|l|}{ Type of Surgical Intervention } \\
\hline Total Thyroidectomy & $96(97.9 \%)$ \\
\hline Completion Thyroidectomy & $2(2 \%)$ \\
\hline Lymph node dissection & 19(19.3\%) \\
\hline Parathyroid reimplantation & $15(15.3 \%)$ \\
\hline \multicolumn{2}{|l|}{ Pathology } \\
\hline Malignant & $41 / 98(41.8 \%)$ \\
\hline Papillary & 39 (95\%) \\
\hline Follicular & $1(2.4 \%)$ \\
\hline Medullary & $1(2.4 \%)$ \\
\hline Albumin (g/dL) & $3.56 \pm 0.03$ \\
\hline Pre-op calcium (mg/dl) & $9.45 \pm 0.06$ \\
\hline Post-op calcium (mg/dl) & $8.20 \pm 0.06$ \\
\hline Decrease in calcium mg/dl; (\%) & $-1.29 \pm 0.12(-15.7 \%)$ \\
\hline iPTH (pg/ml) & $52.2 \pm 5.97$ \\
\hline
\end{tabular}

BMI: Body Mass Index; iPTH: Intraoperative PTH Levels; Shown are number of observations ( $n$ ), percentage values, and means and SEM.

Table 2: Demographic and biochemical characteristics of patients that develop hypocalcemia following thyroidectomy.

\begin{tabular}{|l|l|l|l|}
\hline & Normocalcemia $\mathbf{( n = 7 6 )}$ & Hypocalcemia $\mathbf{( n = 2 2 )}$ & P \\
\hline Age & $62.9 \pm 1.6$ & $44.4 \pm 3.4$ & $<0.0001$ \\
\hline BMI ( kg/m $)$ & $29.2 \pm 0.6$ & $24.9 \pm 0.9$ & 0.0045 \\
\hline iPTH ( pg/ml) & $61.7 \pm 6.2$ & $15.5 \pm 2.4$ & 0.0014 \\
\hline Preoperative calcium (mg/dl) & $9.51 \pm 0.10$ & $9.29 \pm 0.15$ & 0.28 \\
\hline Postoperative calcium (mg/d) & $8.42 \pm 0.05$ & $7.42 \pm 0.06$ & $<0.0001$ \\
\hline Change in calcium (mg/dl) & $-1.05 \pm 0.12$ & $-1.94 \pm 0.12$ & 0.003 \\
\hline Albumin (g/dl) & $3.57 \pm 0.03$ & $3.55 \pm 0.07$ & 0.96 \\
\hline
\end{tabular}

Shown are the number of observations $(n)$, mean values \pm SEM, and the $P$ values. Student $t$ test was employed to probe for differences between groups. BMI: Body Mass Index; iPTH: Intraoperative PTH Levels.

22 hypocalcemic patients, $16(72.7 \%)$ had serologic evidence of hypocalcemia with a corrected serum calcium $\leq 7.5 \mathrm{mg} / \mathrm{dL}$, while 6 (27.2\%) developed symptoms without serologic evidence of hypocalcemia. The average decrease in serum calcium in the normocalcemic group was $1.05 \pm 0.13$, and $1.94 \pm$ 0.12 in the hypocalcemic group (Table 2).
The mean iPTH level for the patients that developed hypocalcemia was $15.5 \pm 2.4 \mathrm{pg} / \mathrm{ml}$, while it averaged $61.7 \pm$ $7.3 \mathrm{pg} / \mathrm{ml}$ for the patients that remained normocalcemic ( $p$ $=0.0003$ ) (Table 2). A statistically significant positive correlation was found between iPTH and postoperative calcium level $(r=0.362$ [Cl 0.17-0.53]; $p=0.0003)$. The ROC-AUC for iPTH 
Citation: Rafols M, Treitl D, Rosario LE, et al. (2020) Predictors of Postoperative Hypocalcemia after Total Thyroidectomy: The Role of a Single Intraoperative Rapid PTH. J Surgical Endocrinol 2(1):61-66

to discriminate PoHC averaged 0.825 [95\% Cl 0.74-0.93], $\mathrm{p}<$ 0.0001 . A cutoff iPTH level of $<16.75 \mathrm{pg} / \mathrm{ml}$ showed a sensitivity of $77.0 \%$, a specificity of $75.0 \%$, a NPV of 92.1 , a PPV of $48.6 \%$, and a Youden index of 0.52 . A cutoff iPTH level of $\geq 36.25 \mathrm{pg} / \mathrm{ml}$ predicted normocalcemia with a specificity of $95.5 \%$ and a PPV $97.8 \%$. Only only 1 of the 22 hypocalcemic patients had an $\mathrm{IPTH}$ value above $36.25 \mathrm{pg} / \mathrm{ml}$.

The average age for normocalcemic patients was $62.9 \pm$ 1.6 years, and $44.4 \pm 3.4$ years for hypocalcemic patients $(p=$ 0.0001 ). The average BMI for the normocalcemic group was $29.2 \pm 0.6 \mathrm{~kg} / \mathrm{m}^{2}$ and $24.9 \pm 1.0$ for the hypocalcemic patients $(p=0.0045)$ (Table 2$)$. Both age and BMI were independently and positively associated with postoperative serum calcium levels $(r=0.406, p<0.0001$; and $r=0.355$, $p: 0.0005$, respectively). Age above 66.5 years and BMls above $29.1 \mathrm{~kg} / \mathrm{m}^{2}$ were each $90 \%$ specific in ruling out hypocalcemia. iPTH was not significantly correlated with age $(r=0.03, p=0.742)$ or $\mathrm{BMI}$ $(r=0.034 ; P=0.75)$, nor was age associated with BMI. Inclusion of the three variables, $\mathrm{iPTH}$, age, and $\mathrm{BMI}$, in the analysis significantly improved the discriminative power for predicting postoperative calcium levels. The ROC-AUC averaged 0.957 [0.91 - 0.99] (Figure 1). Additionally, hypocalcemia was predicted in all patients with $\mathrm{iPTH}<12.5 \mathrm{pg} / \mathrm{ml}$, younger than < 31 years of age, and with a BMI $<22.5 \mathrm{~kg} / \mathrm{m}^{2}$. Performance of any type of lymph node dissection, intraoperative complications, histologic diagnosis, and parathyroid reimplantation was not associated with calcium, iPTH, age, or BMI.

\section{Discussion}

One of the deterrents to a thyroidectomy being an outpatient or short-stay procedure is post-operative monitoring for hypocalcemia. Serial phlebotomy for serum calcium levels can be cumbersome and expensive for patients. Selection of patients at risk for postoperative hypoparathyroidism is essential if surgeons are to be successful at safely discharging thyroidectomy patients on postoperative day 0 or the morning after and limit unnecessary laboratory costs. Identification of these patients during surgery would allow for stratification of patients that are high or low-risk for the development of PoHC and allow for early implementation of calcium supplementation, thus potentially avoiding prolonged hospital stays [13].

Our findings support previous observations indicating that PTH predicts the risk of PoHC. We report that iPTH is positively and significantly associated with postoperative calcium levels. Patients who did not develop PoHC had iPTH levels that were 4 times higher than those who developed hypocalcemia. The American Thyroid Association (ATA) guidelines propose that patients with a postoperative $\mathrm{PTH}$ of $<15 \mathrm{pg} / \mathrm{mL}$ should be considered for oral vitamin $D$ and Calcium supplementation [14]. We found that IPTH levels of PoHC patients averaged $15.5 \pm 2.4 \mathrm{pg} / \mathrm{ml}$, and the optimal ROC-generated cutoff value for iPTH $<16.75 \mathrm{pg} / \mathrm{ml}$ predicted hypocalcemia with a sensitivity of $77.0 \%$ and a specificity of $75 \%$. These findings suggest that iPTH patients with $<16.75 \mathrm{pg} / \mathrm{ml}$ would require serial calcium checks, and/or vitamin $D$ and calcium supplementation. Yet, nearly $25 \%$ of these patients may not develop PoHC. Of interest, if the ROC analysis was used to predict normocalcemia and values of $\mathrm{iPTH} \geq 36.25 \mathrm{pg} / \mathrm{ml}$ predicted normocalcemia with a sensitivity of $95.5 \%$ and a PPV 97.8. Therefore, patients with $\mathrm{iPTH} \geq 36.25 \mathrm{pg} / \mathrm{ml}$ could be monitored with less frequent calcium serology, or none at all. The results of our study will allow stratification of post-thyroidectomy patients into high or low-risk groups with the use

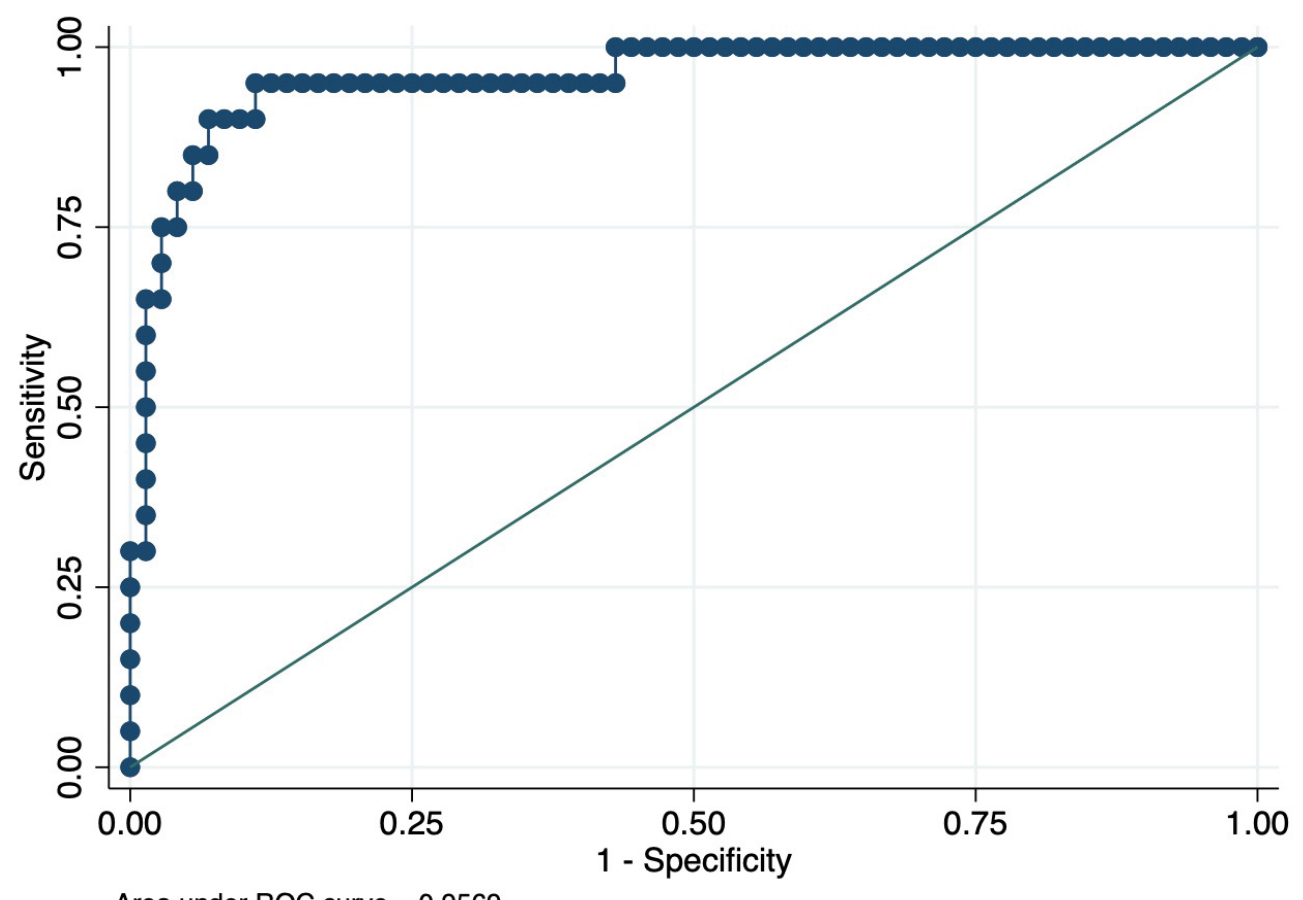

Figure 1: Area under the curve for the receptor operated characteristics (iPTH, age and BMI) in predicting hypocalcemia. 
Citation: Rafols M, Treitl D, Rosario LE, et al. (2020) Predictors of Postoperative Hypocalcemia after Total Thyroidectomy: The Role of a Single Intraoperative Rapid PTH. J Surgical Endocrinol 2(1):61-66

of single iPTH obtained intraoperatively 15 min after thyroidectomy. The patients who are at high risk (iPTH $<16.7 \mathrm{pg} /$ $\mathrm{mL}$ ) may either be monitored with serial calcium checks or receive early PO calcium and vitamin D supplementation. Those patients who fall in between $16.7-36.25 \mathrm{pg} / \mathrm{ml}$ should be monitored per our current protocol without calcium supplementation.

In addition to $\mathrm{iPTH}$, additional variables could also play a role in predicting PoHC. Time of PTH and calcium measurements, patients' characteristics (ethnicity, age, gender, and BMI), comorbidities, thyroid pathology, parathyroid reimplantation, histological diagnosis, surgical procedures and complications, lymph node resections were not significantly associated with hypocalcemia [11]. Here, we describe an association between postoperative calcium levels and the patients' age and BMI, with a significantly increased risk of hypocalcemia in younger patients and lower BMls. During our review of the literature, we found no evidence of previous reports showing the role or value of age and $\mathrm{BMI}$ in predicting the risk of PoHC. Our group of hypocalcemic patients was an average of 18 years younger than the normocalcemic patients and their BMls were $4 \mathrm{~kg} / \mathrm{m}^{2}$ lower. Besides, in addition to $\mathrm{iPTH}$, both age, and BMI were found to be independent predictors of postoperative calcium levels. These findings explain the higher discriminative power in predicting postoperative calcium levels observed when cutoffs for iPTH, age, and $\mathrm{BMI}$ were included in the analysis. The ROC-AUC increased to 0.957 (highest possible value 1.00) when the three variables were combined in the regression analysis. Of interest, all patients that met the following three cutoff values, iPTH $<12.5$ $\mathrm{pg} / \mathrm{ml}, \mathrm{BMI}<22.5 \mathrm{~kg} / \mathrm{m}^{2}$ and age $<31$ years developed hypocalcemia. These findings demonstrate the interplay of factors determining the development of PoHC following a TT.

There is conflicting evidence regarding age and the risk of developing PoHC. Some studies suggest that the younger age group is associated with the development of PoHC $[15,16]$, while other studies have demonstrated the converse $[17,18]$. There is not a clear physiologic explanation for these findings nor is there an exact age cut off where the risks of developing hypocalcemia shifts [19]. However, in our study patient older then 66.5 years did not develop PoHC. Larger scale studies looking at national databases like CESQUIP may provide more information on the association of age and PoHC. Based on our study, surgeons may incorporate the association between young age and PoHC in their decision-making process when deciding to supplement calcium or send patients home early.

To our knowledge, there are no prior publications to suggest that patients with lower BMI were independently associated with PoHC following TT. Although there is evidence to support that thyroidectomy on obese patients is safe, several studies have reported longer operative times [20,21]. One would expect that the added difficulty of performing TT on obese patients would perhaps increase the likelihood parathyroid trauma and PoHC. However, in our cohort, we found that patients with lower BMI have higher risks of developing PoHc. Alternatively, the parathyroid glands of obese patients are often surrounded by more adipose tissue, which may offer a protective factor. Also, differences in calcium and PTH metabolism in obese patients may explain this finding. One study looking at obese patients before bariatric surgery and another looking at biochemical predictors of metabolic syndrome in obese patients both noted that PTH levels were higher in obese patients and positively correlated with body weight $[22,23]$. However, in our study we found no association between $\mathrm{BMI}$ and $\mathrm{PTH}$. Bone is the calcium reserve of the body and the available stores depend on both dietary calcium intake, absorption, and bone density which are increased in patients with higher BMI $[24,25]$. We postulate that thicker cortical bone may be associated with a larger calcium reserve and perhaps be a protective factor for PoHC. Nonetheless, the association between $\mathrm{BMI}$ and $\mathrm{PoHC}$ appears multifactorial and is yet to be elucidated clearly in the post-thyroidectomy population.

\section{Conclusions}

We addressed the utility of a single iPTH level in predicting hypocalcemia after TT. Marked differences in IPTH levels were observed between patients who developed and did not develop PoHC. A ROC analysis was performed to identify what cutoff defines the risk of hypocalcemia with the greatest sensitivity and specificity. A cutoff of iPTH of $<16.7$ was associated with the greatest risk of $\mathrm{PoHC}$, while a cutoff value of $>36.25$ was most associated with normocalcemia. The addition of age and BMI to $\mathrm{IPTH}$, increased the predictive value of $\mathrm{iPTH}$, with a greater risk of $\mathrm{PoHC}$ in younger and leaner patients. All patients with $\mathrm{iPTH}<12.5 \mathrm{pg} / \mathrm{ml}, \mathrm{BMl}<22.5 \mathrm{~kg} / \mathrm{m}^{2}$ and age $<31$ years developed hypocalcemia. Patients could then be divided into high-risk and low-risk subgroups accordingly. High-risk patients should be monitored closely and have a low threshold for oral calcium supplementation, and those determined to be low-risk can receive tailored postoperative calcium monitoring and potentially be discharged the day of surgery. Stratification of risks could help in reducing clinically significant hypocalcemia, delay in discharge, unnecessary lab draws, and reduce costs.

\section{Clinical Significance}

Post-operative hypocalcemia is a commonly encountered complication following total thyroidectomy, typically occurring 24-48 hours after surgery. A single Intraoperative iPTH, age, and BMI can be used as early predictors of PoHC and will facilitate appropriate risk stratification, early calcium supplementation, and potentially avoid extended hospitalizations.

\section{Acknowledgments}

We thank the members of the Department of Surgical Oncology and the surgical residents at Mount Sinai Medical Center Miami Beach, FL for their contribution to managing patients during their hospital stay.

\section{References}

1. Chindavijak S (2007) Prediction of hypocalcemia in postoperative total thyroidectomy using single measurement of intra-operative parathyroid hormone level. J Med Assoc Thail 90(6): 1167-1171.

2. Smink DS (2015) Schwartz's principles of surgery, $10^{\text {th }}$ edn. Ann Surg 261: 1026 . 
3. Cranshaw IM, Moss D, Whineray KE, et al. (2007) Intraoperative parathormone measurement from the internal jugular vein predicts post-thyroidectomy hypocalcaemia. Langenbeck's Arch Surg 392: 699-702.

4. Lee DR, Hinson AM, Siegel ER, et al. (2015) Comparison of intraoperative versus postoperative parathyroid hormone levels to predict hypocalcemia earlier after total thyroidectomy. Otolaryngol Head Neck Surg 153: 343-349.

5. Ritter K, Elfenbein D, Schneider DF, et al. (2015) Hypoparathyroidism after total thyroidectomy: Incidence and resolution. $J$ Surg Res 197: 348-353.

6. Castro A, del Rio L, Gavilan J (2018) Stratifying the risk of developing clinical hypocalcemia after thyroidectomy with parathyroid hormone. Otolaryngol Head Neck Surg 158: 76-82.

7. Lo CY, Luk JM, Tam SC (2002) Applicability of intraoperative parathyroid hormone assay during thyroidectomy. Ann Surg 236: 564-569.

8. Islam MS, Sultana T, Paul D, et al. (2012) Intraoperative serum parathyroid hormone level is an indicator of hypocalcaemia in total thyroidectomy patients. Bangladesh Med Res Counc Bull 38: 84-89.

9. Reddy AC, Chand G, Sabaretnam M, et al. (2016) Prospective evaluation of intra-operative quick parathyroid hormone assay as an early predictor of post thyroidectomy hypocalcaemia. Int J Surg 34: 103-108.

10. Richards ML, Bingener CJ, Pierce D, et al. (2003) Intraoperative parathyroid hormone assay and parathyroid reoperations. Surgery 134: 1049-1055.

11. Mathur A, Nagarajan N, Kahan S, et al. (2018) Association of parathyroid hormone level with postthyroidectomy hypocalcemia a systematic review. JAMA Surg 153: 69-76.

12. Inversini D, Rausei S, Ferrari CC, et al. (2016) Early intact PTH (iPTH) is an early predictor of postoperative hypocalcemia for a safer and earlier hospital discharge: An analysis on 260 total thyroidectomies. Gland Surg 5: 522-528.

13. Noordzij JP, Lee SL, Bernet VJ, et al. (2007) Early prediction of hypocalcemia after thyroidectomy using parathyroid hormone: An analysis of pooled individual patient data from nine observational studies. J Am Coll Surg 205: 748-754.
14. Orloff LA, Wiseman SM, Bernet VJ, et al. (2018) American thyroid association statement on postoperative hypoparathyroidism: Diagnosis, prevention, and management in adults. Thyroid 28: 830-841.

15. Bhattacharyya N, Fried MP (2002) Assessment of the morbidity and complications of total thyroidectomy. Arch Otolaryngol Head Neck Surg 128: 389-392.

16. Cayo AK, Yen TWF, Misustin SM, et al. (2012) Predicting the need for calcium and calcitriol supplementation after total thyroidectomy: Results of a prospective, randomized study. Surgery 152: 1059-1067.

17. Erbil Y, Barbaros U, Temel B, et al. (2009) The impact of age, vitamin $D(3)$ level, and incidental parathyroidectomy on postoperative hypocalcemia after total or near total thyroidectomy. Am J Surg 197: 439-446.

18. Erbil Y (2007) Predictive value of age and serum parathormone and vitamin D3 levels for postoperative hypocalcemia after total thyroidectomy for nontoxic multinodular goiter. Arch Surg 142: 1182.

19. Salinger EM, Moore JT (2013) Perioperative indicators of hypocalcemia in total thyroidectomy: The role of vitamin $D$ and parathyroid hormone. Am J Surg 206: 876-882.

20. Milone M, Musella M, Conzo G, et al. (2016) Thyroidectomy in high body mass index patients: A single center experience. Int J Surg 28: S38-S41.

21. Finel JB, Mucci S, Branger F, et al. (2014) Thyroidectomy in patients with a high BMI: A safe surgery? Eur J Endocrinol 171: 99105.

22. Hamoui N, Anthone G, Crookes PF (2004) Calcium metabolism in the morbidly obese. Obes Surg 14: 9-12.

23. Rueda S, Fernández-Fernández $C$, Romero F, et al. (2008) Vitamin $\mathrm{D}, \mathrm{PTH}$, and the metabolic syndrome in severely obese subjects. Obes Surg 18: 151-154.

24. Heaney RP (2006) Bone as the calcium nutrient reserve. In: Weaver CM, Heaney RP, Calcium in human health. Humana Press, Totowa, NJ , 7-12.

25. Shapses SA, Riedt CS (2006) Bone, body weight, and weight reduction: What are the concerns? J Nutr 136:1453-1456.

DOI: $10.36959 / 608 / 449$

Copyright: (c) 2020 Rafols M, et al. This is an open-access article distributed under the terms of the Creative Commons Attribution License, which permits unrestricted use, distribution, and reproduction in any medium, provided the original author and source are credited. 